\title{
A New U-Net based License Plate Enhancement Model in Night and Day Images
}

\author{
${ }^{1}$ Pinaki Nath Chowdhury, ${ }^{2}$ Palaiahnakote Shivakumara, ${ }^{3}$ RamachandraRaghavendra, \\ ${ }^{1}$ Umapada Pal, ${ }^{4}$ Tong Lu and ${ }^{5}$ Michael Blumenstein \\ ${ }^{1}$ Computer Vision and Pattern Recognition Unit, Indian Statistical Institute, Kolkata, India. \\ Email: pinakinathc@gmail.com, umapada@isical.ac.in. \\ ${ }^{2}$ Faculty of Computer Science and Information Technology, University of Malaya, Kuala Lum- \\ pur, Malaysia. Email: shiva@um.edu.my \\ ${ }^{3}$ Faculty of Information Technology and Electrical Engineering, IIK, NTNU, Norway, \\ raghavendra.ramachandra@ntnu.no \\ ${ }^{4}$ National Key Lab for Novel Software Technology, Nanjing University, Nanjing, China. \\ Email: lutong@nju.edu.cn \\ ${ }^{5}$ Faculty of Engineering and Information Technology, University of Technology, Sydney, \\ Australia. Email: Michael.Blumenstein@uts.edu.au
}

\begin{abstract}
A new trend of smart city development opens up many challenges. One such issue is that automatic vehicle driving and detection for toll fee payment in night or limited light environments. This paper presents a new work for enhancing license plates captured in limited or low light conditions such that license plate detection methods can be expanded to detect images at night. Due to the popularity of Convolutional Neural Network (CNN) in solving complex issues, we explore U-Net-CNN for enhancing contrast of license plate pixels. Since the difference between pixels that represent license plates and pixels that represent background is too due to low light effect, the special property of $U$ Net that extracts context and symmetric of license plate pixels to separate them from background pixels irrespective of content. This process results in image enhancement. To validate the enhancement results, we use text detection methods and based on text detection results we validate the proposed system. .. Experimental results on our newly constructed dataset which includes images captured in night/low light/limited light conditions and the benchmark dataset, namely, UCSD, which includes very poor quality and high quality images captured in day, show that the proposed method outperforms the existing methods. In addition, the results on text detection by different methods show that the proposed enhancement is effective and robust for license plate detection.
\end{abstract}

Keywords:Text detection, License plate detection, U-Net, Convolutional neural networks, Low quality license plate detection.

\section{Introduction}

The era of smart city development for developing countries poses several research challenges. One such challenge is to develop systems for automatic driving, transportation, delivering goods and toll fee regardless of day and night. In this work, we fo- 
cus on license plate detection in images captured at night, low light or limited light conditions [1]. This is essential for making toll fee systems automatic for 24 hours, especially for Malaysia and India to accommodate high-density traffics. There are several powerful methods for license plate detection and recognition in literature [2, 3]. However, most of the methods work well for images captured in day but not night and low light. Due to the lack of sufficient light illumination, the captured images suffer from low contrast, loss of visibility, blur, noise and the effect of non-uniform street light, headlight and moonlight illumination as shown sample images in Fig.Fig.1(a), where it can be seen the effects of the above threats.
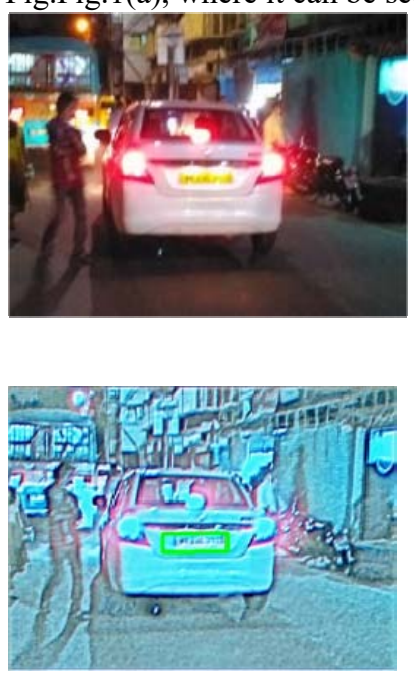
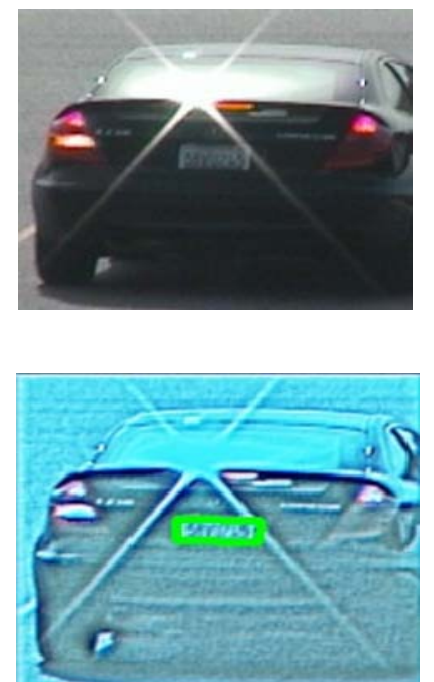

(b) Text detection (EAST) for the proposed enhanced night and day license plate imag-

es

It is noted from the literature that there are many sophisticated methods for license plate detection and recognition for day images. For example, the method in [1] is used for detecting dirty license plate images for high speed applications, the method in [2] is proposed for end to end car license plate detection and recognition with a deep neural network, which focuses on detecting accurate license plates by fixing exact bounding boxes, and the method in [3] deals with an efficient license plate recognition system using convolutional neural networks, which addresses the challenge of low light images. However, as mentionedabove, these methods work well for day images with good contrast but not night images that suffer from poor quality. It is evident from the illustration shown in Fig.1, where one can notice that for the input night and day images with poor quality shown in Fig.1(a), the text detection methodin [4], which explores a powerful deep learning model for text detection in natural scene images, does not detect text for the input images, while for the enhanced images, the same text detection method detects texts well. This observation motivates us to propose a new method for enhancement such that the same text detection or license plate detection methods can be used for license plate detection in night images. 


\section{Related Work}

This section reviews the methods for license plate detection in low quality images and the methods of image enhancement. Asif et al. [5] proposed multinational vehicle license plate detection in complex backgrounds. Rather than dealing with license plates directly, the method finds rare lights as regions of interest, which include license plates. It proposes a heuristic energy map based on vertical edge information for regions of interest. Dense edge areas are extracted for detecting license plates in images. Xie et al. [6] proposed a new $\mathrm{CNN}$ based method for multi-directional car license plate detection. The method presents an idea for addressing the challenges caused by different rotations. Therefore, the method explores $\mathrm{CNN}$ for detecting license plates by estimatingdegree of rotation. Yuan et al. [7] proposed a robust and efficient method for license plate detection. The method explores a line density filter approach for extracting candidate regions, which significantly reducesarea of localization of license plates. The method used the combination of a cascaded classifier and support vector machines for eliminating false candidate regions. Li et al. [2] proposed an end-to-end car license plate detection and recognition with deep neural networks. Instead of developing two separate models for detection and recognition, the method proposed a unified model for both detection and recognition in license plate images. It is noted from the review on theselicense plate detection methods that the methods consider images with high quality (day images) because the main target of the methods is to achieve the best results for license plate detection irrespective of challenges posed by day images. This shows that the scope is limited today images but not low quality and night images.

There are also methods which aim at addressing the challenges of low quality images for license plate detection. Shemarry et al. [8] proposed an ensemble of adaboost cascades of 3L-LBPs classifiers for license plate detection with low quality images. The method combines adaboost classifiers and local binary patterns based features for detecting license plate regions. The method is tuned for addressing issues of low contrast, dusk, dirt, and fogy. Lin et al. [3] proposed an efficient license plate detection system using convolutional neural networks. The method extracts conventional features such as histogram oriented gradients based features and then an SVM classifier for license plate detection in low quality images. Panahi et al. [1] proposed accurate detection and recognition of dirty vehicle plate numbers for high speed applications. To solve the issues of day and night images, the method proposes adaptive thresholding for gray images. The method finds initially key points for images, with these key points it then detects license plate regions through adaptive thresholding. Since thresholds are determined based on empirical results, the method may not be effective for different applications and datasets. In the light of the above discussions on license plate detection of low quality images, it is not sure how the methods work for night or 
low light images, where one can expect not only poor quality but also low contrast, blur, and noises.

There are several methods developed for image enhancement in the past. For instance, Dong et al. [9] proposed a fast-efficient algorithm for enhancement of low lighting video. The method explores the idea of de-hazing for enhancing details of images. Jiang et al. [10] proposed night video enhancement using improved dark channel prior. The method uses an improved dark channel prior model and integrates it with local smoothing and image Gaussian pyramid operators. Sharma et al. [11] proposed contrast enhancement using a pixel-based image fusion in wavelet domain. The method proposes wavelet decomposition to obtain high-frequency sub-bands. Rui and Guoyu [12] proposed a medical X-ray image enhancement method based on TVHomomorphic filter. The method explores the total variation model as transfer function, which balances the brightness in images by increasing high frequency coefficients in Fourier domain. Ravishankar et al. [13] proposed acoustic image enhancement using Gaussian and Laplacian pyramids. The method uses the combination of Gaussian and Laplacian pyramid levels with histogram equalization for enhancing noisy images. Raghunandan et al. [14] proposed a Riesz fractional based model for enhancing license plate detection and recognition. The method focuses on improving edge strength to improve the visibility of low contrast images. Zhang et al. [15] proposed a new fusion-based enhancement for text detection in night video footage. The method combines color information in spatial domain and high-frequency coefficients in frequency domain for enhancing information. It is noted from the above license plate detection methods and enhancement methods that none of the methodscan provide reasonable detection accuracy for night images.

Hence, in this work, we propose a new method based on the U-Net-CNN for enhancing fine details in night images such that license plate detection performance improves. Inspired by the deep learning model proposed for complex medical image segmentation, we explore the same for exploiting the exclusive property of U-Net for image enhancement in this work. It is true that text exhibits symmetrical patterns, such as the uniform color of character pixels, constant stroke width for characters in license plates, the uniform character size, and the uniform spacing between characters. These patterns are invariant to rotation, scaling, color, illumination effect and contrast variations because the patternsare unique, which do not depend on individual pixel values. To extract such context and symmetric patterns, we propose U-Net as it is a special property of $U-N e t[16]$.

\section{Proposed Model}

The proposed work consists of two sub-sections, namely, exploring U-Net for image enhancement and text detection methods to validate the output of image enhancement. As mentioned in the previous section, U-Net helps us to extract context and symmetrical properties of text information. The training of U-Net network is done using an adversarial loss from the EAST network. Therefore, the EAST network along with its loss function becomes the objective function or the empirical risk function for the 
enhancement network. Further, the proposed method is optimized using Adam Optimization technique. Hence intuitively we can expect the enhancer network to modify low-quality images in such a way that the task of text detection becomes easier for algorithms like EAST. To verify the results of U-Net, we propose to use the EAST method [4] which works based on deep learning for text detection in complex natural scene images. The block diagram of the proposed work is presented inFig.2, where we can notice the architecture of U-Net and the enhancement results.

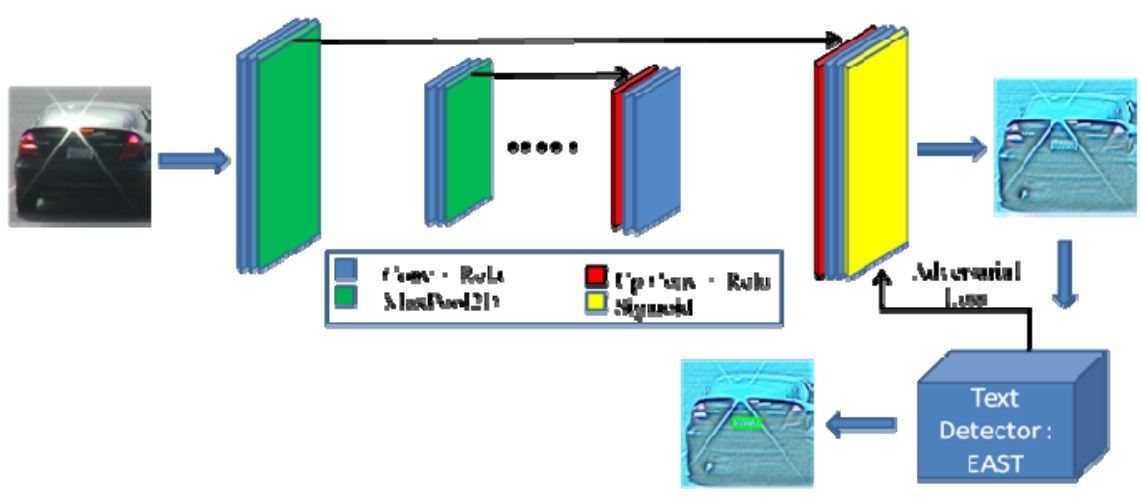

Fig.2. Proposed architecture for the enhancement (a) Text detection (EAST) for the input night and day license plate images

\subsection{The Proposed U-Net based Enhancement Model}

Motivated by the method [16] where U-Net has been proposed for medical image segmentation, we explore the same property with modifications for enhancing images in this work. The architecture used in [16] uses a single input channel, while the proposed U-Net uses three-channel input. The process of U-Net can be described as follows. Let us assume the encoder-decoder to be represented by a function $\boldsymbol{f}_{\text {unet }}$ and the network parameters be represented by $\boldsymbol{\theta}_{\text {unet. }}$. We first evaluate:

$$
Z_{i}=f_{\text {unet }}\left(X_{i}, \theta_{\text {unet }}\right)
$$

where $\boldsymbol{X}_{\boldsymbol{i}}$ is the input image, $\boldsymbol{Z}_{\boldsymbol{i}}$ is the output image, $f_{\text {unet }}$ is the function whichrepresents the encoder-decoder network, $\theta_{\text {unet }}$ is the parameters.As mentioned before, the entire EAST network and its loss function is the objective function for UNet like enhancement network. If $f_{\text {EAST }}$ represents EAST network along with its loss function, then we would expect $f_{\mathrm{EAST}}\left(\mathrm{Z}_{\mathrm{i}}\right)$ to be a scalar value (as we mentioned that $f_{\text {EAST }}$ not only represents the EAST network but also its loss function which essentially always returns a scalar value).

Hence the loss function for the enhancement network can be represented by the following equation:

$$
\text { Loss }=f_{\text {EAST }}\left(f_{\text {unet }}\left(X_{i}, \theta_{\text {unet }}\right)\right)
$$

From equation (2) we know the optimization function, which we want to solve in order to find the optimal $\theta_{\text {unet }}^{*}$ as shown below: 


$$
\theta_{\text {unet }}^{*}=\underset{\theta_{\text {unet }}}{\operatorname{argmin}}\left(f_{\text {EAST }}\left(f_{\text {unet }}\left(X_{i}, \theta_{\text {unet }}\right)\right)\right)
$$

The U-Net architecture used two convolution layers followed by a Max Pooling layer, which essentially performs the down-sampling operation. The activation function Rectified Linear Unit is used after each convolution layer. Initializations of all the convolution parameters are done using He normal initializer.The network was trained using a learning rate of 0.0001 given as the input to Adam Optimizer, batch size of 7 and trained for 27000 steps. We observe that near around 17000 steps the enhancement network starts to give decent results. As done in U-Net architecture, we perform concatenation operation of up-sampling layers with their corresponding downsampling layers. The output is a 3-channel matrix of the same shape as that of the input and sigmoid activation function is used.
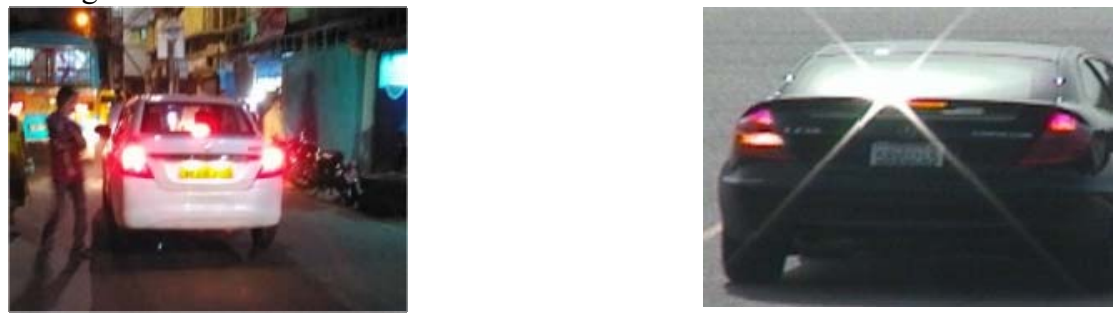

(a) Input night and day license plate images
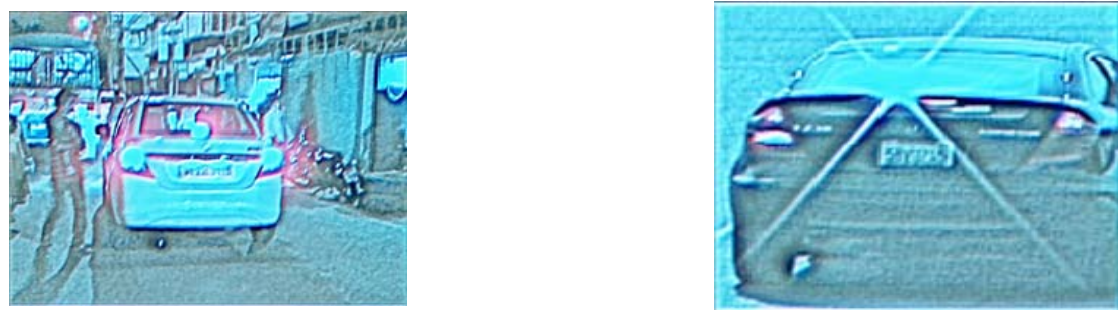

(b) The result of the proposed enhancement model

Fig.3. The effectiveness of the proposed enhancement model

The U-Net integrates the local information through down sampling path and up sampling path, which results in extracting context information (pattern). Since the kernel used in U-Net is independent of input image size, the model does not need dense layer and works well for any size of input images [16]. The effect of the proposed U-Net for image enhancement is shown in Fig.3, where it is seen that for the poor quality images affected by lights in case of nigh image and non-uniform illumination effect in case of day image as shown in Fig.3(a), the proposed model enhances text details as shown in Fig.3(b). It is observed from Fig.3(b) that one can guess the presence of texts compared to the texts in input images. This is the advantage of the proposed U-Net. 


\subsection{Text Detection for the Enhancement Images}

To validate the output of the previous step, we implement the state-of-the-art methods, which address almost all the challenges of text detection in natural scene images, such as low contrast, complex background, multi-script, and orientation. If a text detection method detects texts correctly for an enhanced image, it is saidthat the proposed enhancement steps are effective and useful. The EAST method [4] proposes an efficient and accurate scene text detector, which explores a single neural network.The main reason to choose the above method is that itis addressing the issues which are part of license plate detection in night images. In addition, the codes of the method are available publicly for supporting research reducibility. Sample license plate detection for the input and output of the proposed enhancement is shown in Fig.4(a) and Fig.4(b), respectively. It is observed from Fig.4 that the EAST method does not detect any text in the input images, while for the enhanced image, the same method detects the license plate, accurately. Therefore, we can argue that the proposed enhancement is effective for both night images and day images with poor quality.
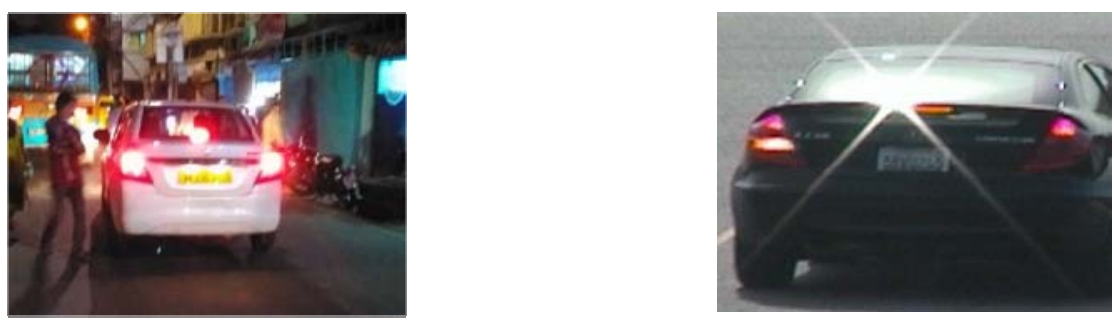

(a) Text detection (EAST) for night day license plate images before enhancement
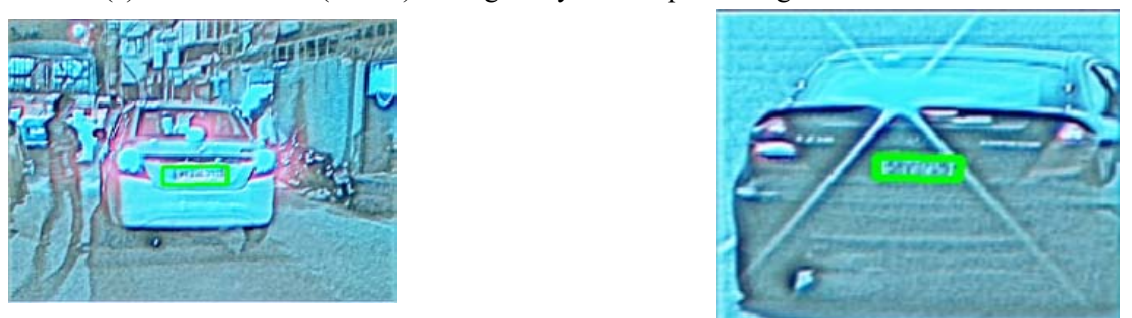

(b) Text detection (EAST) for the night and day license plate images after enhancement

Fig.4. Validating the enhancement results through text detection performance.

\section{$4 \quad$ Experimental Results}

To evaluate the proposed enhancement model, we create a new dataset by capturing license plate images in night, low light and limited light conditions. This dataset includes 200 images for experimentation with a large variation, such as low contrast, poor quality, affected lights, and multiple vehicles in images affected by headlights of vehicles, etc. At the same time, we also test the proposed enhancement model on the 
benchmark dataset called UCSD [17], which includes low quality as well as high quality images to show that the proposed method is effective for enhancing not only night images but also day images affected by poor quality. The UCSD dataset is further divided into three sub-categories, namely, UCSD-1 which consists of images with blur and low-resolution images, UCSD-2 which consists of images of severe blur with noises, and UCSD-3 which comprises images with good focus and quality. The details of our dataset and the benchmark dataset are reported in Table 1. In summary, the proposed enhancement model is evaluated on both night and day images to show that the proposed enhancement is useful for improving license plate detection performance.

Table 1. The details of our dataset and benchmark datasets of license plate images

\begin{tabular}{|c|c|c|c|c|}
\hline Dataset & Night-Our-LPR & Day-LPR UCSD-1 & Day-LPR UCSD-2 & Day-LPR UCSD-3 \\
\hline Testing & 200 & 1290 & 114 & 291 \\
\hline \multirow{2}{*}{ Resolution } & Min: 2067x2066 & Min: $463 \times 685$ & Min: 241x241 & Min: 480x640 \\
& Max: $3120 \times 4160$ & Max: $463 \times 685$ & Max: 241x241 & Max: 480x640 \\
\hline Blur & YES & YES & YES & NO \\
\hline Script & YES & NO & NO & NO \\
\hline $\begin{array}{c}\text { Imbalanced } \\
\text { Illumination }\end{array}$ & YES & NO & NO & NO \\
\hline Orientation & YES & NO & NO & NO \\
\hline Perspective & YES & NO & NO & NO \\
\hline
\end{tabular}

To measure the performance of the enhancement, we use quality measures, namely, BRISQUE (Blind/Reference Less Image Spatial Quality Evaluator) which measures naturalness and smoothness of images, and NRIQA (Blind No Reference Image Quality Assessment) which measures degradations caused by blur, noise and distortion artifacts. The definitions and more explanation can be found in [18], where the quality measure is defined. The reason to choose these two measures for evaluating the proposed enhanced results is that unlike MLE, PSNR measures, these two do not require ground truth for measuring the quality of enhanced images. In addition, these two measures smoothness (fine details of the image) and degradations affected by illumination, distortion, noise, etc. The low value of BRISQUE indicates an image is enhanced, while a high value of NRIQA indicates better quality. Similarly, for measuring text detection results, we use standard metrics, namely, Recall (R), Precision (P) and F-measure (F). We calculate these measures before enhancement and after enhancement to assess the contribution of the enhancement model. In case of before enhancement experiments, text detection methods consider input images directly for text detection. In case of after enhancement, text detection methods consider the enhanced images as input for text detection.

To show the objectiveness of the proposed method, we implement three enhancement methods to compare with the results of the proposed enhancement model in terms of quality measures. Jiang et al. [10] proposed night video enhancement using improved dark channel prior, which explores color values in spatial domain for en- 
hancement.Rui and Guoyu [12] proposed a medical X-ray image enhancement method on TV homomorphic filter, which explores frequency coefficients in frequency domain for enhancement. Zhang et al. [15] proposed a new fusion-based enhancement for text detection in night video footage, which combines both spatial and frequency domain-based features for enhancement. The reason to choose the above methods for comparative study is that the objective of all the methods was similar to the objective of the proposed work. For text detection experiments, we use codes of EAST [4] and PixelLink [19], which explore different deep learning models for text detection in natural scene images. The reason to choose these methods for validating the proposed enhanced results is that the methods are robust and addressed almost all the challenges of text detection in natural scene images.
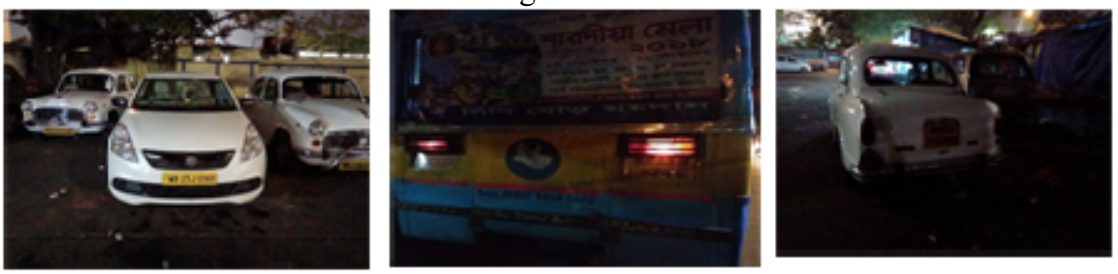

(a) Input night license plate images our dataset

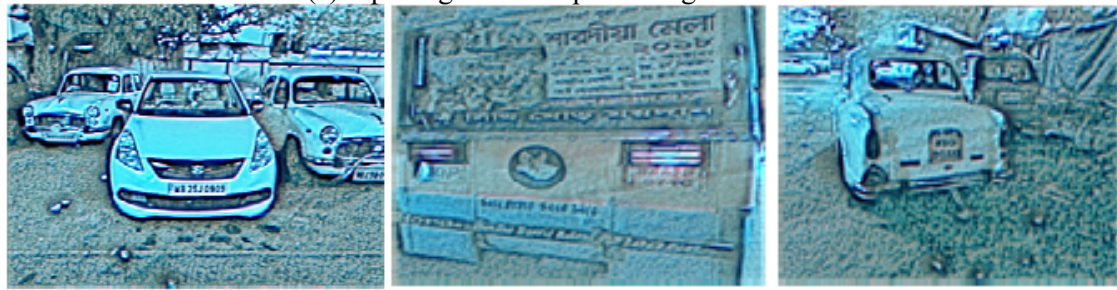

(b) The result of the proposed enhancement model for night images
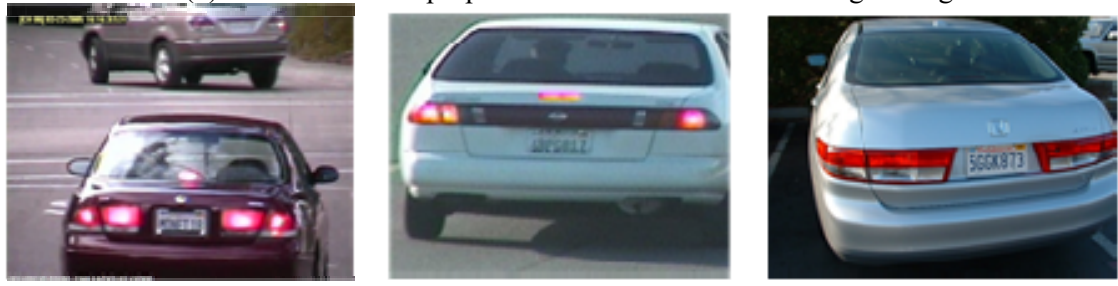

(c) Input day low quality license plate images of different datasets.
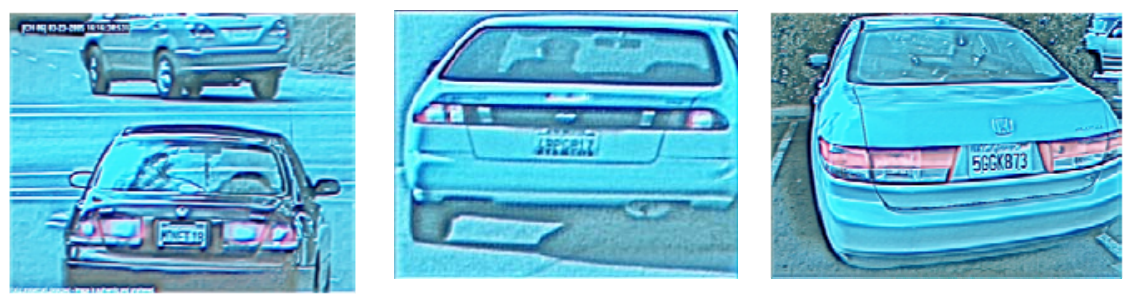

(d) The result of the proposed enhancement model for different day low quality images. Fig.5. Sample results of the proposed enhancement model for day and night datasets. 


\subsection{Evaluating the Proposed Enhancement Model}

Qualitative results of the proposed enhancement for both night and day images are shown in Fig.5, where it is noted from the input images shown in Fig.5(a) and Fig.5(c) that it is hard to read the number plates. On the other hand, Fig.5(b) and Fig.5(d) show that the number platesare visible compared to those in the input images. Therefore, one can assert that the proposed enhancement is robust, and can improve license plate detection performance for night and poor quality images. Quantitative results of the proposed and existing methods are reported in Table 2 for our night and three standard datasets. Table 2 shows that the BRISQUE measure is low for the proposed enhancement compared to the results of before enhancement as well as the existing methods for all the datasets. The low score of BRISQUE indicates the quality of images is improved compared to before enhancement. Similarly, the NRIQE is high for the proposed enhancement compared to the results of before enhancement and the existing methods for all datasets. The higher score of NRIQE indicates that the quality is improved compared to before enhancement. It is expected that the existing methods should give low BRISQUE and high NRIQE after enhancement compared to before enhancement. But due to the limitations of the existing methods, the results are not consistent for all the four datasets. On the other hand, the proposed enhancement is consistent for all the four datasets in terms of both BRISQUE and NRIQE. This is because of the advantages of U-Net, which extracts context and symmetric patterns from the text. Therefore, we can conclude that the proposed enhancement is robust not only for night images but also day images with poor quality.

Table 2. The performance of the proposed and existing enhancement models before and after enhancement on our UCSD-1, UCSD-2 and UCSD-3 datasets

\begin{tabular}{|c|c|c|c|c|c|c|c|c|}
\hline Datasets & \multicolumn{2}{|c|}{ Our-Night } & \multicolumn{2}{c|}{ UCSD-1 } & \multicolumn{2}{c|}{ UCSD-2 } & \multicolumn{2}{c|}{ UCSD-3 } \\
\hline Measures & BRISQUE & NRIQE & BRISQUE & NRIQE & BRISQUE & NRIQE & BRISQUE & NRIQE \\
\hline Before & 37.99 & 3.27 & 46.01 & 3.59 & 37.06 & 5.12 & 45.74 & 3.80 \\
\hline Proposed & 21.48 & 5.48 & 44.85 & 6.98 & 33.80 & 8.32 & 29.29 & 4.49 \\
\hline Jinag et al. [10] & 40.56 & 3.37 & 54.79 & 3.94 & 68.34 & 4.91 & 39.72 & 2.41 \\
\hline Rui and Guoyu [12] & 39.77 & 3.57 & 54.43 & 4.35 & 74.32 & 5.16 & 40.95 & 3.18 \\
\hline Zhang et al. [15] & 37.52 & 3.17 & 52.28 & 3.75 & 64.18 & 4.39 & 35.52 & 2.31 \\
\hline
\end{tabular}

\subsection{Validating the Proposed Enhancement Model Through Text Detection}

To test the usefulness of the proposed enhancement compared to the existing enhancement methods, we conduct text detection experiments for the outputs of the proposed and existing enhancement methods on all the four datasets. Qualitative results of the EAST and PixelLink text detection methods for the input and enhanced images of all the four datasets are shown in Fig.6(a)-Fig.6(d). It is noted from Fig.6 that the text detection methods do not detect textsaccurately in the input images as shown in Fig.6(a) and Fig.6(b). This indicates that the input imagesare complex and suffer from poor quality and other adverse factors. However, the results in Fig.6(b) and Fig.6(d) show that the same methods detect almost all the texts in the input imag- 
es correctly. Therefore, it is concluded that the proposed enhancement is effective and useful for improving text detection performance for night images and day image with poor quality.
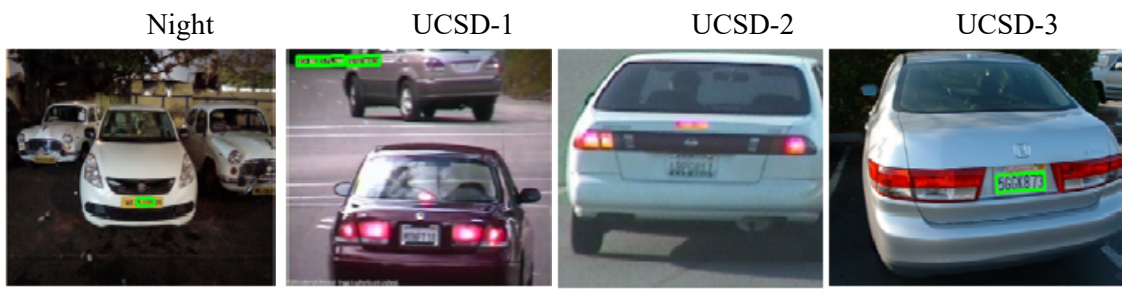

(a) The text detection result of the EAST method before enhancement.
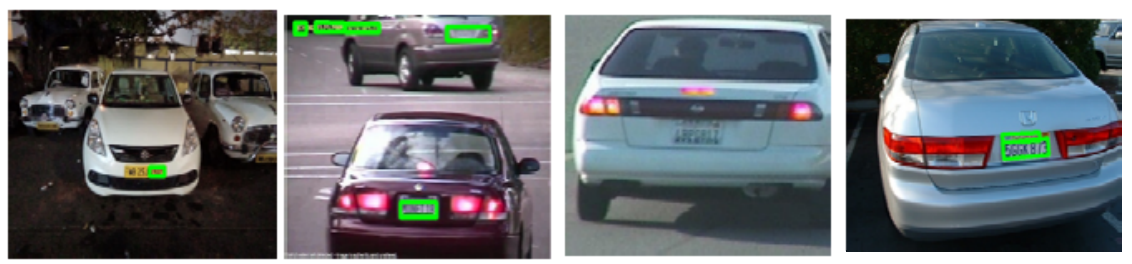

(b) The text detection result of the PixelLink method before enhancement.
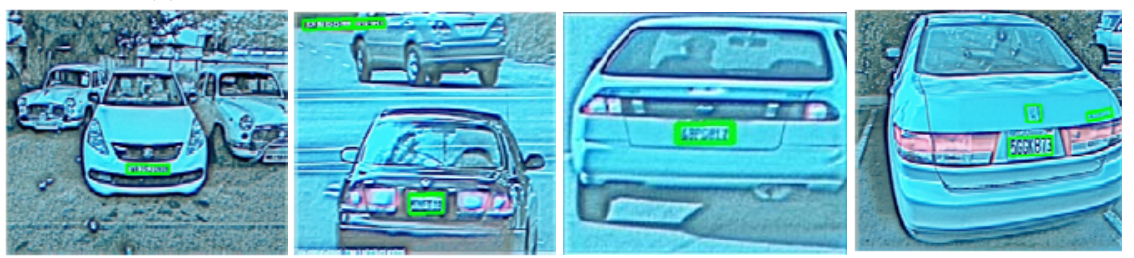

(c) The text detection result of the EAST method after enhancement.
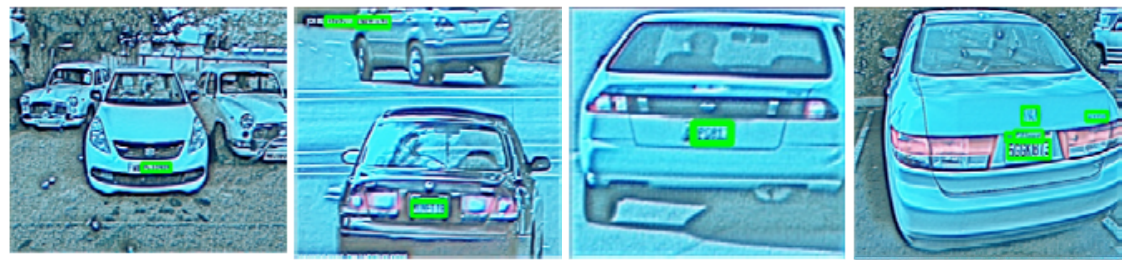

(d) The text detection result of the PixelLink method after enhancement.

Fig.6. Text detection performance before after enhancement.

Quantitative results of text detection methods before and after enhancement for all the four datasets are reported in Table 3-Table 6, respectively. It is observed from Table 3-Table 6 that the proposed enhancement is the best at F-measure calculated according to text detection methods compared to the existing enhancement methods as well as before enhancement for all the four datasets. However, due to limitations of the existing enhancement methods, text detection results are not consistent for enhanced images compared to before enhancement and the proposed enhancement. 
There is a significant improvement in the text detection result after enhancement compared to before enhancement and the existing methods. With this analysis, we can argue that the proposed enhancement is effective and useful for improving text detection performance for night and day with poor quality images.

Table 3. Text detection performance on the proposed and existing enhancement results before and after enhancement for our dataset

\begin{tabular}{|c|l|l|l|l|l|l|}
\hline \multirow{2}{*}{ Methods } & \multicolumn{3}{|c|}{ EAST [8] } & \multicolumn{3}{c|}{ PixelLink [9] } \\
\cline { 2 - 7 } & $\mathrm{P}$ & $\mathrm{R}$ & $\mathrm{F}$ & $\mathrm{P}$ & $\mathrm{R}$ & $\mathrm{F}$ \\
\hline Before Enhancement & 0.971 & 0.260 & 0.414 & 0.939 & 0.237 & 0.379 \\
\hline Jinag et al. [10] & 0.972 & 0.266 & 0.417 & 0.986 & 0.272 & 0.426 \\
\hline Rui and Guoyu [12] & 0.953 & 0.234 & 0.376 & 0.969 & 0.422 & 0.588 \\
\hline Zhang et al. [15] & 0.986 & 0.274 & 0.429 & 0.953 & 0.431 & 0.594 \\
\hline Proposed Method & 0.929 & 0.279 & $\mathbf{0 . 4 3 0}$ & 0.952 & 0.454 & $\mathbf{0 . 6 1 5}$ \\
\hline
\end{tabular}

Table 4. Text detection performance on the proposed and existing enhancement results before and after enhancement for UCSD-1 dataset

\begin{tabular}{|c|c|c|c|c|c|c|}
\hline \multirow{2}{*}{ Methods } & \multicolumn{3}{|c|}{ EAST [8] } & \multicolumn{3}{c|}{ PixelLink [9] } \\
\cline { 2 - 7 } & $\mathrm{P}$ & $\mathrm{R}$ & $\mathrm{F}$ & $\mathrm{P}$ & $\mathrm{R}$ & $\mathrm{F}$ \\
\hline Before Enhancement & 1.0 & 0.328 & 0.494 & 1.0 & 0.386 & 0.557 \\
\hline Jinag et al. [10] & 1.0 & 0.305 & 0.467 & 1.0 & 0.420 & 0.592 \\
\hline Rui and Guoyu [12] & 1.0 & 0.281 & 0.439 & 0.991 & 0.430 & 0.600 \\
\hline Zhang et al. [15] & 1.0 & 0.293 & 0.453 & 0.982 & 0.434 & 0.602 \\
\hline Proposed Method & 1.0 & 0.343 & $\mathbf{0 . 5 1 1}$ & 1.0 & 0.432 & $\mathbf{0 . 6 0 3}$ \\
\hline
\end{tabular}

Table 5. Text detection performance on the proposed and existing enhancement results before and after enhancement for UCSD-2 dataset

\begin{tabular}{|c|c|c|c|c|c|c|}
\hline \multirow{2}{*}{ Methods } & \multicolumn{3}{|c|}{ EAST [8] } & \multicolumn{3}{c|}{ PixelLink [9] } \\
\cline { 2 - 7 } & $\mathrm{P}$ & $\mathrm{R}$ & $\mathrm{F}$ & $\mathrm{P}$ & $\mathrm{R}$ & $\mathrm{F}$ \\
\hline Before Enhancement & 1.0 & 0.588 & 0.740 & 1.0 & 0.519 & 0.683 \\
\hline Jinag et al. [10] & 1.0 & 0.352 & 0.521 & 0.985 & 0.666 & 0.795 \\
\hline Rui and Guoyu [12] & 1.0 & 0.539 & 0.700 & 0.8 & 0.627 & 0.703 \\
\hline Zhang et al. [15] & 0.969 & 0.313 & 0.474 & 0.897 & 0.686 & 0.777 \\
\hline Proposed Method & 0.933 & 0.823 & $\mathbf{0 . 8 7 4}$ & 0.813 & 0.813 & $\mathbf{0 . 8 1 3}$ \\
\hline
\end{tabular}

Table 6. Text detection performance on the proposed and existing enhancement results before and after enhancement for UCSD-3 dataset 


\begin{tabular}{|c|l|l|l|l|l|l|}
\hline \multirow{2}{*}{ Methods } & \multicolumn{3}{|c|}{ EAST [8] } & \multicolumn{3}{c|}{ PixelLink [9] } \\
\cline { 2 - 7 } & P & R & F & P & R & F \\
\hline Before Enhancement & 0.989 & 0.375 & 0.544 & 1.0 & 0.391 & 0.562 \\
\hline Jinag et al. [10] & 1.0 & 0.386 & 0.557 & 1.0 & 0.396 & 0.562 \\
\hline Rui and Guoyu [12] & 1.0 & 0.375 & 0.546 & 1.0 & 0.419 & 0.590 \\
\hline Zhang et al. [15] & 1.0 & 0.379 & 0.550 & 1.0 & 0.416 & 0.588 \\
\hline Proposed Method & 1.0 & 0.395 & $\mathbf{0 . 5 6 6}$ & 0.990 & 0.421 & $\mathbf{0 . 5 9 1}$ \\
\hline
\end{tabular}

Table 7. Robustness Performance on the proposed and existing enhancement results before and after enhancement for our dataset by randomly resizing images

\begin{tabular}{|c|c|c|l|l|l|}
\hline Methods & \multicolumn{2}{|c|}{ Text Enhancement } & \multicolumn{3}{c|}{ Text Detection using EAST [8] } \\
\hline & BRISQUE & NRIQE & P & R & \multicolumn{1}{|c|}{ F } \\
\hline $\begin{array}{c}\text { Before En- } \\
\text { hancement }\end{array}$ & 37.99 & 3.27 & 0.971 & 0.257 & 0.406 \\
\hline Jing et al[10] & 21.48 & 5.48 & 0.972 & 0.265 & 0.416 \\
\hline $\begin{array}{c}\text { Rui and } \\
\text { Guoyu [12] }\end{array}$ & 40.56 & 3.37 & 0.953 & 0.234 & 0.375 \\
\hline $\begin{array}{c}\text { Zhang et } \\
\text { al[15] }\end{array}$ & 39.77 & 3.57 & 0.986 & 0.273 & 0.427 \\
\hline $\begin{array}{c}\text { Proposed } \\
\text { Method }\end{array}$ & 37.52 & 3.17 & 0.929 & 0.277 & $\mathbf{0 . 4 2 7}$ \\
\hline
\end{tabular}

\section{$5 \quad$ Conclusion and Future Work}

In this work, we have proposed a new method for enhancing license plate image captured at night, low light and limited light conditions. The proposed method explores the special property of U-Net, which extracts context and symmetric patterns with the minimum number of samples, for enhancing fine details of license plates in images. In case of text or number plates, one can expect the uniform color, and symmetric patters such as constant stroke width of characters and regular spacing between characters. To verify enhancement results, we have performed text detection on enhanced imagesand on the original input images to test the contribution of the proposed enhancement. Text detection results on our own night license plate images and day images with poor quality show that the proposed enhancement method is better compared to the existing enhancement methods. However, when we look at number plates in enhanced images, it is not easy to recognize characters by OCR. Therefore, our plan is to extend the work for improving recognition results in the near future. 


\section{References}

1. R. Panahi and I. Gholampour, "Accurate detection and recognition of dirty vehicle plate numbers for high speed applications", IEEE Trans. ITS, 18, pp 767-779, 2017.

2. H. Li, P. Wang and C. Shen, "Toward End to end car license plate detection and recognition with deep neural networks", IEEE Trans. ITS, 20, pp 1126-1136, 2019.

3. C. H. Lin, Y. S. Lin and W. C. Liu, "An efficient license plate recognition system using convolutional neural networks", In Proc. ICASI, pp 224-227, 2018.

4. X. Zhou, C. Yao, H. Wen, Y. Wang, S. Zhou, W. He, and J. Liang, "East: an efficient and accurate scene text detector," in Proc. CVPR, pp. 2642-2651, 2017.

5. M. R. Asif, Q. Chun, S. Hussain, M. S. Fareed and S. Khan, "Multinational vehicle license plate detection in complex backgrounds", Journal of Visual Communication and Image Representation, 46, pp 176-186, 2017.

6. L. Xie, W. Ahmad, L. Jin, Y. Liu and S. Zhang, "A new CNN based method for multidirectional car license plate detection", IEEE Trans. ITS, 19, pp 507-517, 2018.

7. Y. Yuan, W. Zou, Y. Zhao, X. Wang, X. Hu and N. Komodakis, "A robust and efficient approach to license plate detection", IEEE Trans., IP, 26, pp 1102-1114, 2017.

8. M. S. A. Shemarry, Y. Li and S. Abdulla, "Ensemble of adaboost cascades of 3L-LBPs classifiers for license plated detection with low quality images, ESWA, 92, pp 216-235, 2018.

9. X. Dong, G. Wang, Y. Pang, W. Li, J. Wen, W. Meng and Y. Lu, "Fastefficient algorithm for enhancement of low lighting video", In Proc. ICME, 20111, pp. 1-6.

10. X. Jiang, H. Yao, S. Zhang, X. Lu and W. Zeng, "Night video enhancement using improved dark channel prior", In Proc. ICIP, 2013, pp 553-557.

11. S. Sharma, J. J. Zuo and G. Fang, "Contrast enhancement using pixel based image fusion in wavelet domain”, In Proc. FC3I, 2016, pp 285-290.

12. W. Rui and W. Guoyu, "Medical X-ray image enhancement method based on TVHomomorphic filter", In Proc. ICIVC, 2017, pp 315-318.

13. P. Ravishankar, R. S. Sharmila and V. Rajendran, "Acoustic image enhancement using Gaussian and Laplacian pyramid-a multiresolution based technique", Multimedia Tools and Applications, 2018, pp 5547-5561.

14. K. S. Raghunandan, P. Shivakumara, H. A. Jalab, R. W. Ibrahim, G. H. Kumar, U. Pal and T. Lu, "Riesz fractional based model for enhancing license plate detection and recognition”, IEEE Trans. CSVT, 28, pp 2276-2288, 2018.

15. C. Zhang, P. Shivakumara, M. Xue, L. Zhu, T. Lu and U. Pal, "New fusion based enhancement for text detection in night video footage", In Proc. PCM, 2018, pp 46-56.

16. O. Ronneberger, P. Fischer and T. Brox, "U-Net Convolutional Networks for Biomedical Image Segmentation”, In Proc. MICCAI, Springer, LNCS, Vol. 9351, pp 234-241, 2015.

17. A. Zamberletti, I. Gallo and L. Noce, "Augmented text character proposals and convolutional neural networks for text spotting from scene images", In Proc. ACPR, pp 196-200, 2015.

18. V. Khare, P. Shivakumara, A. Kumar, C. C. Chan, T. Lu and M. Blumenstein, "A quad tree based method for blurred and non-blurred video text frame classification through quality measures", In Proc. ICPR, pp 4012-4017, 2016.

19. D. Deng, H. Liu, X. Li and D. Cai, "PixelLink: Detecting scene text via instance segmentation", In Proc. AAAI, 2018. 